\title{
Case Report of Giant Cell Tumor of Proximal Tibia
}

\author{
Dr. Rajesh Ambulgekar ${ }^{1}$, Dr. Pritesh Kothari ${ }^{2}$, Dr. Prajyot kandolkar ${ }^{3}$, \\ Dr. Rohit Thakkar ${ }^{4}$ \\ Department of Orthopedics, Dr Shankarrao Chavan Goverment Medical College, Nanded
}

\begin{abstract}
Middle aged old female with swelling in left knee suggestive of giant cell tumor was treated with excisional biopsy with curettage, high speed burr drilling, phenol cauterisation and cementing. Sample sent for histopathology was consistent with diagnosis of giant cell tumour. Patient started weight bearing from second postoperative day. No recurrence has been seen after 1 year of follow up.
\end{abstract}

Keywords: Bone cement, Giant cell tumour, proximal tibia, Excisional biopsy, phenol

\section{Introduction}

Giant cell tumour is seen in $4 \%$ of all bone tumours and it represents $10 \%$ of all malignant bone tumours. Occurs in age group between 20 to 55 years. More common in females. Its activity ranges from borderline to malignant lesion. It is locally aggressive and destructive lesion . Tumour is notorious for high recurrence rates after excision. Since tumour has high recurrence rates after it is treated by combination of various modalities available to reduce the recurrence rates. There is evidence in the literature which suggests that tumour treated by multiple modalities of treatment has a lower recurrence rates than a tumour treated by single modality. Here is one such case of giant cell tumour treated by multiple adjuvant therapies. After 12 months of follow up no recurrence has been seen. Tumour arises from the epiphyses of long bones. Most common sites are proximal tibia, distal femur, and distal radius. Radiographycally it seen as expansile exophytic mass.

\section{Case report}

40 years old female presented with history of pain and swelling in left knee for 3 months. There was no history of trauma or fever .On examination there was a firm non tender well defined swelling arising from

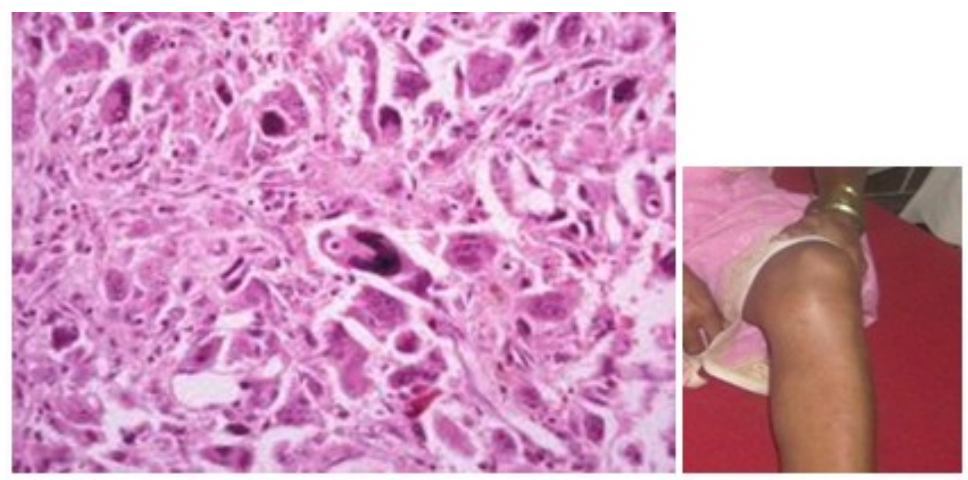

Microscopic picture

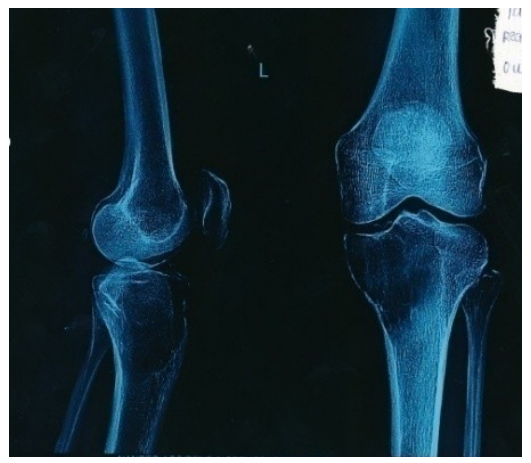

Preop X ray 


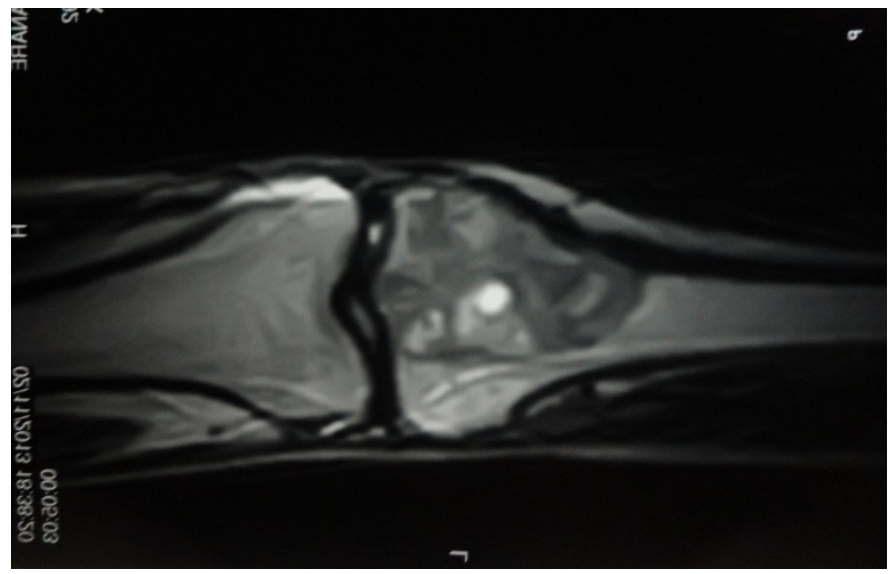

MRI picture

proximaltibia. There was no crepitus no tenderness and knee range of movement was normal. On x ray eccentric epiphyseal exophytic mass was seen in proximal aspect of left tibia. There was no join invasion seen on $\mathrm{x}$ ray. Chest Xray of the patient was normal. MRI revealed $6 * 4 \mathrm{~cm} \mathrm{~cm}$ multiloculated lytic mass on medial aspect of left tibia. FNAC was done showed presence of giant cells. With clinical diagnosis and FNAC of giant cell tumour treatment was planned. Tumour was treated by excisional biopsy and curettage of the margins of lesion. Margins were cauterised using phenol. Resulting cavity that was formed was filled with bone cement polymethylmethacrylate. Sample was sent for histopathological examination Microscopy was consistent with the diagnosis of giant cell tumour. Multinucleated giant cells with oval or round shaped stromal cells were seen. There was no evidence of wound infection in postoperative period. Patient was discharged after suture removal. Patient could comfortably bear weight on second postoperative day.

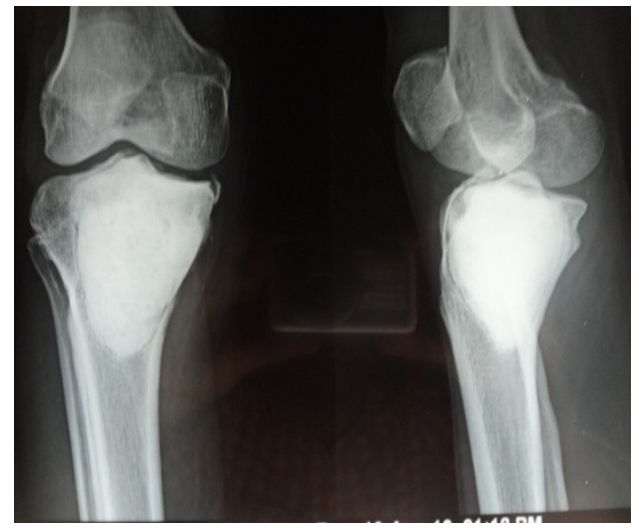

She resumed her routine physical activity after discharge. Patient was followed up every month for first 3 months and thereafter every 3 month. X ray of knee was taken on every follow up to see for any evidence of recurrence. No recurrence has been seen on first 12 months of follow up.

\section{Discussion}

Giant cell tumour represents $4 \%$ of all bone tumours of bone. Tumour is primarily benign but has tendency to turn malignant. Tumour is notorious for recurrences. Tumour arises from the epiphysis of bone, Tumour grows as a expansile exophytic mass.[1,5.10] Tumour is grey to reddish brown in colour and is composed of soft vascular friable tissue. Microscopically it consists of multinucleated giant cells scattered in vascularised network of proliferating round, oval or spindle shaped cells surrounding by indistinct cytoplasm. $\{5,10]$ For this reason there are various modalities of treatment available for tumour to prevent the recurrences .[5] Various modalities available for treatment are excision of tumour followed by curettage, wide local excision, burr drilling, other adjuvant therapies like phenol cauterisation, cryotherapy, intralesional chemotherapeutic agents like adriamycin or methotrexate.[5,7,4] Resultant defect that is formed is treated based on location and size of tumour. In case distal ulna, proximal radius, proximal fibula, coccyx, sacrum resection of involved bone is performed.[4,5,7,8] For distal femur , proximal tibia , distal radius bon cement or bone graft or combination is used. For larger tumours around knee joint reconstruction with technique like turn o plasty is used.[1,5,7] Some aggressive and recurrent tumours may require amputation. Chemotherapy and radiotherapy is 
used for unresectable malignant tumours.[1,9] Adjuvant therapies used to reduce recurrences. Phenol cautery and cryotherapy kills malignant cells at the margin of tumour. Bone cement by exothermic reaction exerts a cytotoxic effect on tumour cells. [5,7] Cavity can be filled by bone cement or bone graft. Both methods has its own advantages and drawbacks. Advantages of bone cement are cement exerts thermal effect which kills cells, makes detection of recurrence easier and gives structural support and allows early weight bearing. Drawbacks are damage to articular cartilage when used in subchondral lesions and cement though strong in compression is weak when subjected to shear. Advantages of bone graft are undergoes remodelling along stress lines and once incorporated reconstruction is permanent. Drawbacks are autograft quantity is limited, donor site morbidity, allograft is expensive and recurrence is difficult to identify. [10]

\section{Conclussion}

The main primary treatment of GCT is surgery, the type of which depends on preoperative evaluation which includes clinical evaluation that involves the site and size of the tumour in relation to surrounding structures, together with plain X-ray, CT scan and/or MRI as indicated and tissue biopsy to define tumour grade. Curettage alone results in high rate of local recurrence. On the other hand, curettage and adjuvant procedure like burr drilling, phenol cauterisation, cryosurgery, argon beam etc. using bone cement or bone grafts as filler gives low rate of local recurrence. Resection is recommended for stages IB and IIB, extremely large lesions, and in cases where resection results in no significant morbidity as proximal fibula and flat bones. Amputation is preserved for massive recurrences and malignant transformation.

\section{References}

[1]. Chapter 21: Benign/aggressive tumors of bone," in Campbell's Operative Orthopaedics, T. S. Canale, Ed., vol. 1, pp. 883-886, Mosby, New York, NY, USA, 11th edition, 2007.

[2]. Dahlin DC. Caldwell Lecture. Giant cell tumor of bone: Highlights of 407 cases. AJR Am J Roentgenol.1985, 144: 955-60.

[3]. Dahlin DC, Cupps RE, Johnson EWJr. Giant cell tumor: A study of 195 cases. Cancer. 1970, 25: $1061-70$.

[4]. Campanacci M, Baldini N, Boriani S, Sudanese A.Giant cell tumor of bone. J Bone Joint Surg Am. 1987,69: 106-14.

[5]. Canale ST, Beaty JH: Benign/Aggressive tumors of bone. In Campbell's Operative Orthopaedics 11th edition. Philadelphia: Mosby;2007:883-886.

[6]. Enneking WF. A system of staging musculoskeletal neoplasms. Clin Orthop. 1986, 204: 9-24

[7]. Tunn PU, Schlag PM. Giant cell tumor of bone. An evaluation of 87 patients. Z Orthop Ihre Grenzgeb.

[8]. Doita M, Harada T, Iguchi T, Sumi M, Sha H, Yoshiya S, Kurosaka M. Total sacrectomy and reconstruction for sacral tumors. Spine. 2003, 28 (15): 296-301003, 141 (6): 690-8. Bennett CJ, Marcus RB, Million RR, Enneking W.

[9]. Radiation therapy for giant cell tumor of bone. Int J Radiat Oncol Biol Phys. 1993, 26: 299-304.

[10]. Current concepts in bone and soft tissue tussue tumour By Dr. Ajay Purie and Dr. M. G. Agarwal first edition. 